\title{
Errata
}

\section{Vol. 65, No. 20}

In the $M M W R$ report, "Fatal Abusive Head Trauma Among Children Aged < 5 Years — United States, 1999-2014," multiple errors occurred in the figure on page 507 (corrected text is noted by underline). The top box should have read, "The underlying cause code in the death record must indicate assault as the intent of injury." The bottom left box should have read,
"Abusive head trauma diagnosis codes (S02.0-S02.1, S02.7S02.9, S04.0, S06.0-S06.9, S07.1, S07.8-S07.9, S09.7-S09.8, T90.2, T90.5, T90.8-T90.9)." The bottom right box should have read, "Must exclude all abusive head trauma injury codes," and "Must exclude all of these traumatic brain injury diagnosis codes."

FIGURE. Classification of fatal assault-related traumatic brain injury* with and without abusive head trauma ${ }^{\dagger}$ among children aged $<5$ years — United States, 1999-2014

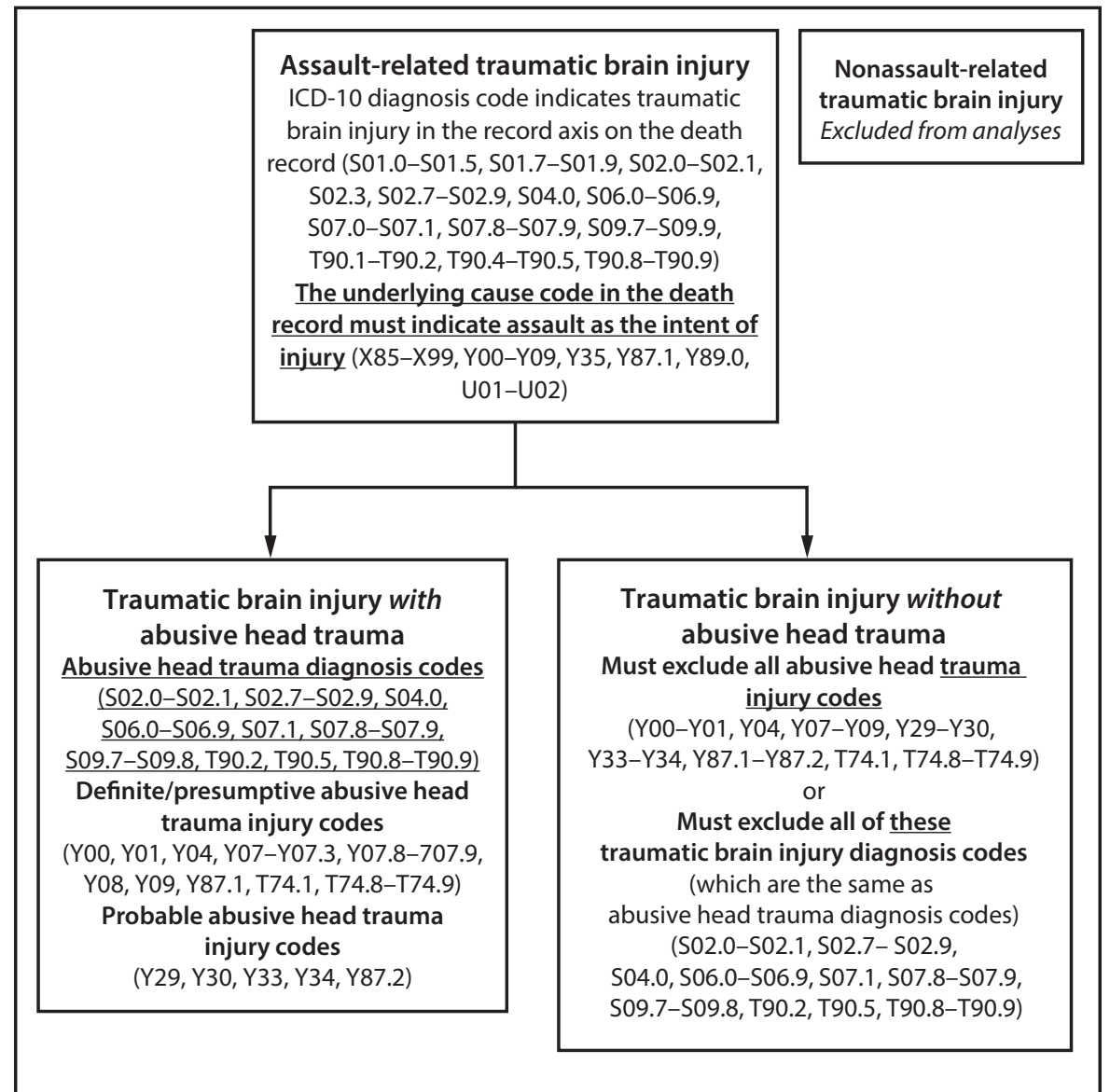

Abbreviation: ICD = International Classification of Diseases.

* Fatal traumatic brain injury is defined as a death caused by a bump, blow, or jolt to the head, or by a penetrating injury that disrupts normal brain function, and includes intentional gunshot wounds and stab wounds. These deaths can be classified as assault-related or nonassault-related.

† Fatal abusive head trauma is defined as a death caused by an injury to the skull or intracranial contents of an infant or child aged $<5$ years attributable to inflicted blunt impact and/or violent shaking, and excludes deaths from injuries resulting from neglectful supervision and deaths from gunshot or stab wounds and penetrating trauma. 